\title{
COMPOST COMO INDUCTOR DE LA SUCESIÓN VEGETAL EN UN ÁREA AFECTADA POR MINERÍA A CIELO ABIERTO EN LA MICROCUENCA DEL RÍO LA VEGA, TUNJA, BOYACÁ
}

\author{
Compost as a means for the ecological succession in an area affected by opencast \\ mining in the La Vega river basin, Tunja, Boyacá
}

\author{
Gilma Elizabeth Gutiérrez Acevedoํㅜㄹ Francisco Cortés Pérez² \& Nydia Liseth Gómez Albarrán ${ }^{3}$
}

Gutiérrez A. G., Cortés, P. F. \& Gómez, A. N. (2015). Compost como inductor de la sucesión vegetal en un área afectada por minería a cielo abierto en la microcuenca del río La Vega, Tunja, Boyacá. Colombia Forestal, 18(2),241-254.

Recepción: 13 de agosto de 2014

\section{RESUMEN}

Se aplicó compost para la inducción de la sucesión vegetal en un área afectada por minería a cielo abierto en la microcuenca del río La Vega. En un diseño experimental completamente aleatorizado se establecieron tres tratamientos en parcelas de $25 \mathrm{~m}^{2}$ usando sustrato estéril y compost en las siguientes proporciones: $T_{1}: 8: 1 ; T_{2}: 4: 1 ; T_{3}: 2: 1$ y un control ( $T_{4}$, sin compost). En cada parcela se siguió la sucesión durante nueve meses, se calcularon los índices de diversidad de Shannon, Simpson y riqueza específica, y se elaboraron ANOVAS de medidas repetidas y análisis de componentes principales para los tratamientos y el control. En total se registraron 15 especies, cinco de ellas de la familia Asteraceae. El análisis de varianza mostró que no existieron diferencias significativas $(p=0.24)$ con respecto a la cobertura, pero sí con respecto a la altura ( $p=0.0001$ ) entre los tratamientos, y que T3 fue el que presentó la mejor respuesta, representada en mayor riqueza específica y diversidad en relación
Aprobación: 1 de febrero de 2015

con los demás tratamientos y el control al final del seguimiento.

Palabras clave: cobertura vegetal, ecología de la restauración, enmienda orgánica, restauración de ecosistemas.

\begin{abstract}
The application of compost to promote ecological succession in an area affected by opencast mining in the La Vega river basin was studied. Three treatment concentrations were established in plots of $25 \mathrm{~m}^{2}$ in a completely randomized experimental design, using sterile deposit and compost in the following ratios; $T_{1}: 8: 1 ; T_{2}: 4: 1 ; T_{3}: 2: 1$ and a control $\left(\mathrm{T}_{4}\right.$, no compost). The bio-diversification in every plot was evaluated during a nine month period. Shannon, Simpson and Species Richness diversity indices were calculated. Repeated measurements of the principle components using ANOVA comparing the treatment to the control were analyzed.
\end{abstract}

1 Grupo de Investigación Ecología de Bosques Andinos Colombianos (EBAC), Universidad Pedagógica y Tecnológica de Colombia. Tunja, Colombia elizabethgacevedo@gmail.com Autor para correspondencia.

2 Grupo de Investigación Ecología de Bosques Andinos Colombianos (EBAC), Universidad Pedagógica y Tecnológica de Colombia. Tunja, Colombia. ecologiadocencia@gmail.com

3 Grupo de Investigación Ecología de Bosques Andinos Colombianos (EBAC), Universidad Pedagógica y Tecnológica de Colombia. Tunja, Colombia.nnidgo_207@hotmail.com 
Fifteen species were recorded, five of them in Asteraceae. The variance analysis showed that there were no significant differences $(p=0.24)$ regarding coverage. With respect to height $(p=0.0001)$, $\mathrm{T}_{3}$ showed the best response in terms of a higher species richness and diversity when compared with the other treatments or the control.

Key Words: vegetation, restoration ecology, organic amendment, ecosystem restoration.

\section{INTRODUCCIÓN}

Las explotaciones mineras a cielo abierto producen efectos negativos sobre los componentes bióticos, abióticos, geomorfológicos y microclimáticos del ecosistema (Lubke \& Avis, 1998; Andrés, 2009; Barrera et al., 2010). A mediana o gran escala, la minería cambia la composición y estructura de los ecosistemas, afecta su productividad, daña los acuíferos, aumenta el vertido de sedimentos a las fuentes hídricas que introducen cambios en los ciclos biogeoquímicos y del agua en los ecosistemas (Villas-Boas \& Page, 2001; Etter et al., 2006; Miranda, 2009; Barrera, 2009; Negrete, 2013).

Estos efectos dificultan el proceso de restablecimiento natural de las áreas explotadas (Bradshaw, 1997) debido a barreras ecológicas como factores limitantes y tensionantes que deja la explotación (Aguilar, 2009; Barrera, 2009; Barrera et al., 2010).

Los elementos más afectados del ecosistema son el suelo, la vegetación y la fauna. En el suelo se presenta acidificación, pérdida de nutrientes y humedad, carencia de espacios para la germinación y crecimiento de la vegetación nativa, por la eliminación del banco de semillas y la escasa o nula concentración de materia orgánica, aspectos que favorecen el aumento de procesos erosivos; si el suelo se elimina completamente, la vegetación no se puede establecer por la baja disponibilidad de agua y los cambios drásticos en la temperatura, y la fauna queda privada de su hábitat, refugio y disponibilidad de alimento (Miranda, 2009; Barrera, 2009; Barrera et al., 2010).

A causa de los cambios ocasionados por la minería a cielo abierto en los ecosistemas, es importante realizar trabajos de ecología de la restauración que brinden conocimientos, metodologías y herramientas para ejecutar estrategias de restauración ecológica que contribuyan a la recuperación de parte de la estructura y función de los sitios explotados, y los conduzcan a un estado similar al ecosistema de referencia (SER, 2004; PNR, 2012).

Una de las estrategias de restauración ecológica es el uso de enmiendas orgánicas, entre estas están los biosólidos (Barrera \& Valdés, 2007; Barrera et al., 2010), utilizados para recuperar la vegetación de las áreas disturbadas por actividades extractivas a cielo abierto en la cantera Soratama (Ochoa \& Barrera, 2007) y la arenera Juan Rey (Rozo, 2007), ubicadas en Bogotá D.C. Otra enmienda orgánica que se puede utilizar es el compost, el cual ayuda en el restablecimiento de coberturas vegetales, mejora el terreno para que se extiendan las raíces y se estabilice el suelo, aumenta la disponibilidad de nutrientes y la temperatura, incrementa la capacidad de regulación hídrica, ya que retiene humedad en época de sequía y reduce la escorrentía en época de invierno, aumenta el contenido de humus y disminuye la perdida de nutrientes, por lo que favorece el establecimiento de vegetación en suelos desnudos y áreas escarpadas producidas por la minería (Kowaljow \& Mazzarino, 2007; Miranda, 2009; Domene et al., 2011).

En consecuencia, la recuperación de la vegetación con ayuda de compost en áreas disturbadas por explotaciones mineras a cielo abierto, es una de las herramientas para mitigar la destrucción de los ecosistemas de la microcuenca, recuperar parte de sus funciones, reducir la ocurrencia de inundaciones y los daños en diversos sectores, en este caso, del municipio de Tunja por el desbordamiento del río La Vega en los periodos de Iluvia. Ante estos problemas ocasionados por la actividad 
minera, se propuso aplicar la mezcla estéril/compost en diferentes proporciones para inducir la sucesión vegetal en un área afectada por minería a cielo abierto en la microcuenca del río La Vega, Tunja, Boyacá.

\section{METODOLOGÍA}

\section{Área de estudio}

El experimento se llevó a cabo en la microcuenca del río La Vega, Ladrillera Fénix, vereda Tras del Alto, al noroccidente del municipio de Tunja, Boyacá, a una altitud de 2839 m, con coordenadas $05^{\circ} 33^{\prime} 40^{\prime \prime}$ de latitud $N$ y $073^{\circ} 22^{\prime} 85^{\prime \prime}$ de longitud W. La temperatura promedio mensual es de $13.8^{\circ} \mathrm{C}$, humedad relativa de $81 \%$ y precipitación promedio anual de $648 \mathrm{~mm}$ (estación meteorológica UPTC (CP), área operativa Boyacá, Casanare).

El montaje experimental se realizó en un área de fácil acceso de aproximadamente $490 \mathrm{~m}^{2}$, con vegetación herbácea en sus alrededores y predominancia de pastos; esta área en breve va a ser utilizada como mina a cielo abierto, por lo que se procedió con maquinaria pesada a retirar toda la vegetación y los primeros $80 \mathrm{~cm}$ de capa superficial, compuesta por suelo y primera capa de estéril; se realizó una nivelación del terreno, que tenía una pendiente aproximada de $15^{\circ}$, que pasó a ser de $5^{\circ}$. Adicionalmente, se trasladó material estéril de la mina en explotación y se mezcló con el compost, que se adquirió en la empresa de servicios públicos del municipio de Sogamoso (Coservicios), y que es obtenido como producto del tratamiento de los residuos orgánicos de plazas, viveros y residuos caseros (Coservicios, 2007). De esta manera se asemejaron las condiciones de una mina a cielo abierto.

\section{Diseño experimental}

El experimento tuvo un diseño completamente al azar, debido a que se eliminó la pendiente como factor de bloqueo. En el diseño se delimitaron 12 parcelas de $5 \times 5 \mathrm{~m}$, separadas $1.5 \mathrm{~m}$ entre sí para evitar la mezcla entre tratamientos y facilitar la toma de datos (Barrera et al., 2010). Los tratamientos consistieron en mezclas estéril/compost $\mathrm{V} / \mathrm{V}$ en diferentes proporciones así: $T_{1} 8: 1, T_{2} 4: 1, T_{3}: 2: 1$ y $\mathrm{T}_{4}$ : control, sin compost, con tres réplicas de cada uno. El estéril es el material sin valor económico que no contiene minerales de valor recuperables y que es necesario remover durante la operación minera para extraer el mineral útil (Ministerio de Minas y Energía, 2003).

\section{Toma de datos}

Para el seguimiento de la sucesión vegetal se establecieron cuadrantes fijos en cada parcela y con ayuda de un marco removible de 1 x $1 \mathrm{~m}$ (Müeller \& Ellenberg, 1974) se tomaron datos de frecuencia y cobertura, además se registró la altura de las plantas teniendo en cuenta los rangos propuestos por Rangel \& Lozano (1986). Después de iniciada la sucesión, el seguimiento se efectuó cada mes durante nueve meses; como los cambios fueron poco notorios, las figuras se hicieron con los datos de cada trimestre.

Para la caracterización físico-química del sustrato se tomó una muestra por tratamiento y el control a una profundidad entre 0 y $20 \mathrm{~cm}$, al inicio y final del seguimiento, que fueron analizadas en el laboratorio de diagnóstico en suelos y agua de la Universidad Pedagógica y Tecnológica de Colombia, donde se evaluaron 21 características: $\mathrm{Mg}$ (magnesio), Textura, pH, Ca (calcio), Ca/Mg, Bray II (fosforo disponible), CICE (capacidad de intercambio de cationes efectiva), A (arena), T ${ }^{\circ}$ (temperatura), \% M.O. (porcentaje de materia orgánica), $\mathrm{K}$ (potasio), K/Mg, CE (conductividad eléctrica), $\mathrm{Na}$ (sodio), L (limo), Ca/K, Ca+Mg/K, Ar (arcilla), Mg/K y Al (aluminio); muestras de compost y de material estéril también fueron analizadas (Tabla 1).

La identificación de las especies se hizo en campo con la ayuda de botánicos especialistas, hasta el nivel taxonómico posible y por medio de 
Tabla 1. Caracterización físico-química del compost y del material estéril, utilizados en el experimento.

\begin{tabular}{|c|c|c|c|}
\hline Característica analizada & Compost & Estéril & $\mathbf{T}_{3} \mathbf{I}$ \\
\hline \multicolumn{4}{|c|}{$\left(\mathrm{meq} / 100 \mathrm{~g}\right.$ de suelo-cmol $\left.{ }^{+} \mathrm{Kg}^{-1}\right)$} \\
\hline$\overline{M g}$ & 3.45 & 1.33 & 3.45 \\
\hline $\mathrm{Ca}$ & 13.9 & 2.06 & 18.1 \\
\hline $\mathrm{Al}$ & 0.0 & 1.0 & 0.0 \\
\hline CICE & 29.3 & 5.19 & 27.8 \\
\hline $\mathrm{K}$ & 10.1 & 0.29 & 5.11 \\
\hline \multicolumn{4}{|c|}{$\left(\mathrm{meq} / 100 \mathrm{~g}-\mathrm{cmol}^{+} \mathrm{Kg}^{-1}\right)$} \\
\hline $\mathrm{K} / \mathrm{Mg}$ & 2.93 & 0.22 & 1.48 \\
\hline $\mathrm{Ca} / \mathrm{Mg}$ & 4.03 & 1.55 & 5.25 \\
\hline $\mathrm{Ca} / \mathrm{K}$ & 1.38 & 7.1 & 3.54 \\
\hline $\mathrm{Ca}+\mathrm{Mg} / \mathrm{K}$ & 1.72 & 11.7 & 4.22 \\
\hline $\mathrm{Mg} / \mathrm{K}$ & 0.34 & 4.59 & 0.68 \\
\hline \multicolumn{4}{|c|}{ Porcentaje (\%) } \\
\hline $\mathrm{Na}$ & 6.38 & 9.83 & 4.34 \\
\hline $\mathrm{MO}$ & 8.84 & 0.22 & 4.52 \\
\hline $\mathrm{N}$ & 0.44 & 0.01 & 0.23 \\
\hline Arena & 63 & 39 & 55 \\
\hline Limo & 26 & 22 & 20 \\
\hline Arcilla & 11 & 39 & 25 \\
\hline Textura & Franco-arenoso & Franco-arcilloso & Franco Ar. arenoso \\
\hline Bray II (ppm) & 170 & 6.86 & 155 \\
\hline $\mathrm{pH}\left[\mathrm{H}_{3} \mathrm{O}\right]^{+}$ & 7.9 & 5.3 & 7.4 \\
\hline Temperatura $\left({ }^{\circ} \mathrm{C}\right)$ & 14 & 14.8 & 15.3 \\
\hline $\mathrm{CE}(\mathrm{ds} / \mathrm{m})$ & 12.2 & 0.52 & 5.42 \\
\hline
\end{tabular}

fotografías se confirmaron en colecciones del herbario de la Universidad Pedagógica y Tecnológica de Colombia y herbarios virtuales de la Universidad Nacional de Colombia y del Missouri Botanical Garden.

\section{Análisis de datos}

La cobertura vegetal de cada parcela del experimento se evaluó teniendo en cuenta los rangos de cobertura basados en el porcentaje de área cubierta por las plantas, propuestas por Braun-Blanquet (1964). Se calcularon los índices de Shannon, Simpson y riqueza específica (Ramírez, 2006).

Se realizaron análisis de varianza (ANOVAS) y pruebas de diferencia mínima significativa (LSD) (Gutiérrez \& De la Vara, 2012) para encontrar divergencias entre tratamientos de acuerdo al porcentaje de cobertura y altura de las especies durante los nueve meses de seguimiento, y se realizó un análisis de componentes principales (ACP) para observar la variación en las características físico-químicas del sustrato al inicio y final del muestreo con el paquete estadístico Statgraphic 5.1. (1980).

\section{RESULTADOS}

Durante el estudio se censaron un total de 15 especies herbáceas pertenecientes a 11 géneros y siete familias, siendo Asteraceae la familia mejor representada con cinco especies, Poaceae con tres especies, Amaranthaceae y Fabaceae con dos especies (Tabla 2).

\section{Diversidad en los tratamientos y el control}

La tendencia general mostró que los tratamientos $\mathrm{T}_{1}, \mathrm{~T}_{2}$ y $\mathrm{T}_{4}$ (control) presentaron alta dominancia, baja abundancia y riqueza específica, mientras $T_{3}$ presentó la mayor diversidad a lo largo del tiempo. 
Tabla 2. Familias y especies vegetales presentes por tratamiento, $\mathrm{T} 1: 8: 1 ; \mathrm{T} 2: 4: 1 ; \mathrm{T} 3: 2: 1$ y control (T4) durante los nueve meses de seguimiento.

\begin{tabular}{|c|c|c|c|c|c|}
\hline Familia & Especie & $\mathrm{T}_{1}$ & $T_{2}$ & $T_{3}$ & Control $\left(T_{4}\right)$ \\
\hline \multirow{5}{*}{ Asteraceae } & Bidens triplinervia Kunth & $\mathrm{x}$ & $\mathrm{x}$ & $\mathrm{x}$ & \\
\hline & Bidens sp. & $\mathrm{x}$ & $\mathrm{x}$ & $\mathrm{x}$ & $\mathrm{x}$ \\
\hline & Hypochaeris radicata $\mathrm{L}$. & & $x$ & & $x$ \\
\hline & Sonchus oleraceus L. & $\mathrm{x}$ & $\mathrm{x}$ & $\mathrm{x}$ & \\
\hline & Sonchus sp. & $\mathrm{x}$ & $\mathrm{x}$ & $\mathrm{x}$ & \\
\hline \multirow{2}{*}{ Amaranthaceae } & Chenopodium petiolare Kunth & $\mathrm{x}$ & $\mathrm{x}$ & $\mathrm{x}$ & \\
\hline & Chenopodium sp. & $\mathrm{x}$ & $\mathrm{x}$ & $\mathrm{x}$ & \\
\hline \multirow{2}{*}{ Fabaceae } & Medicago sp. & $\mathrm{x}$ & $\mathrm{x}$ & & \\
\hline & Acacia decurrens Willd & $\mathrm{x}$ & $\mathrm{x}$ & $\mathrm{x}$ & \\
\hline \multirow[t]{2}{*}{ Malvaceae } & Malva parviflora L. & & & $x$ & \\
\hline & Poa annua L. & $\mathrm{x}$ & $\mathrm{x}$ & $x$ & $\mathrm{x}$ \\
\hline \multirow[t]{2}{*}{ Poaceae } & Poa sp. & & & & $\mathrm{x}$ \\
\hline & Pennisetum clandestinum Hochst. ex Chiov. & $\mathrm{x}$ & $\mathrm{x}$ & $\mathrm{x}$ & $\mathrm{x}$ \\
\hline Polygonaceae & Polygonum aviculare L. & & & $x$ & \\
\hline Plantaginaceae & Plantago linearis Kunth & & & & $\mathrm{x}$ \\
\hline
\end{tabular}
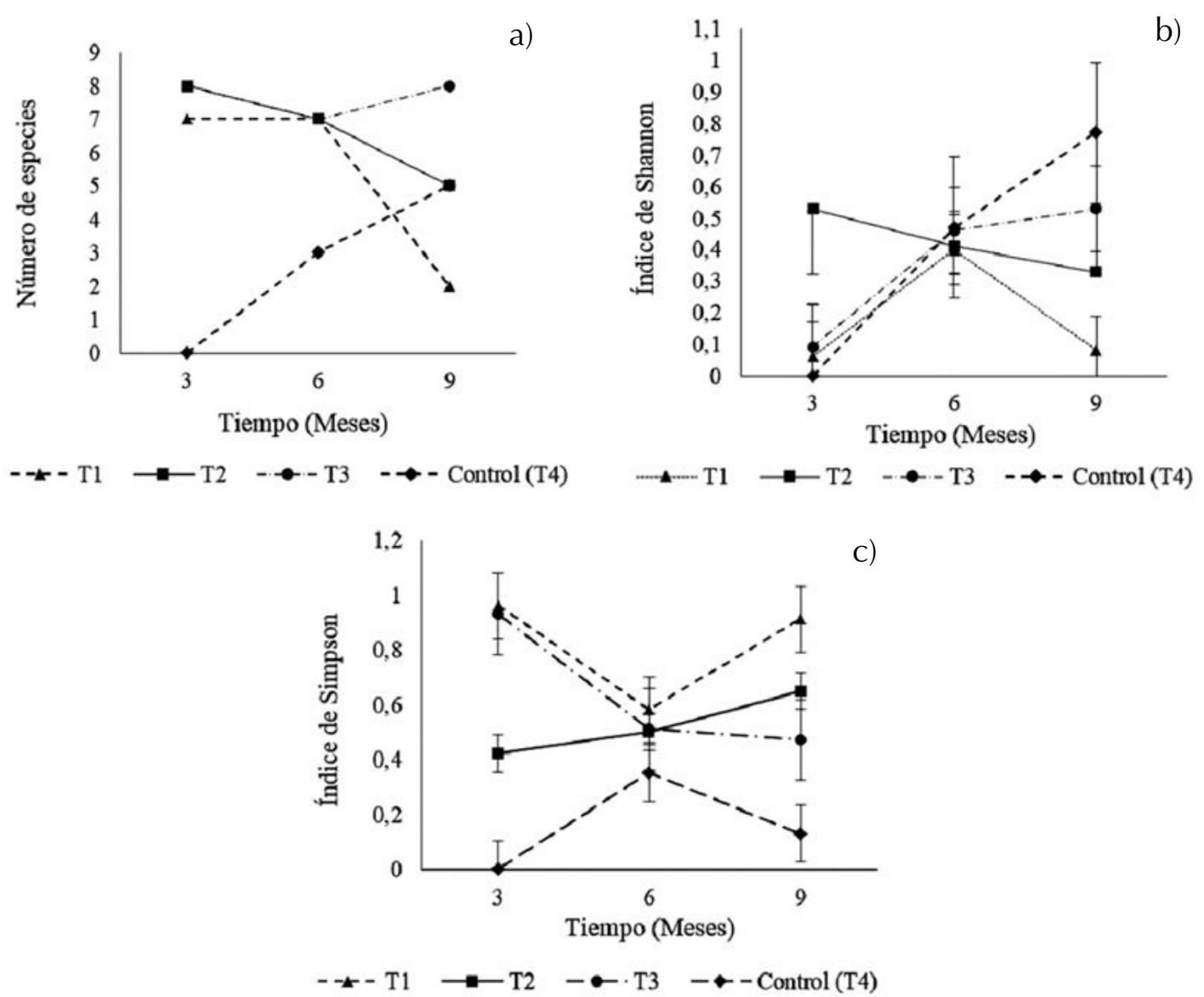

Figura 1. Cambios en los índices de los tratamientos y el control durante el tiempo de seguimiento. a) Riqueza específica, b) Índice de diversidad de Shannon, c) Índice de dominancia de Simpson. 
La riqueza específica (Figura 1a) mostró que $\mathrm{T}_{1}$ presentó siete especies entre los tres y seis meses y disminuyó a dos especies a los nueve meses; $\mathrm{T}_{3}$ presentó ocho especies del inicio al final del muestreo, y el control $\left(\mathrm{T}_{4}\right)$ no registró especies en los primeros tres meses muestreados, sin embargo a los nueve meses presentó cinco especies. En el índice de Shannon (Figura 1b), $T_{2}$ presentó la mayor diversidad de especies a los tres meses y disminuyó su valor hacia el noveno mes; en $\mathrm{T}_{3}$ aumentó la diversidad del tercero al noveno mes y en el control $\left(\mathrm{T}_{4}\right)$ aumentó el valor del índice entre el sexto y noveno mes. El índice de Simpson (Figura 1c) mostró que $T_{3}$ presentó el valor más bajo entre el sexto y noveno mes, mientras $\mathrm{T}_{1} \mathrm{y}_{2}$ presentaron la mayor dominancia en este periodo de tiempo; el control $\left(\mathrm{T}_{4}\right)$ incrementó el valor del índice a los seis meses, pero su dominancia fue la menor entre los seis y nueve meses.

\section{Análisis de varianza (ANOVA) para la cobertura y altura}

No existieron diferencias estadísticas significativas $(p=0.24)$ entre los promedios de cobertura de los tratamientos, durante los nueve meses de seguimiento, sin embargo, la proporción de compost influyó en la recuperación de la cobertura vegetal (Figura 2a). Existieron diferencias muy significativas $(p=0.0001)$ entre los tratamientos con respecto a la altura de las especies durante los nueve meses muestreados. La prueba de diferencia mínima significativa (LSD), con un $95 \%$ de confiabilidad, indicó que existieron diferencias entre los promedios de altura entre los tratamientos y el control durante los meses evaluados, donde sobresale T3 de los demás tratamientos y el control. $T_{1}$ y $T_{2}$ tuvieron promedios de altura semejantes (b, c, d) durante los nueve meses de seguimiento; mientras $\mathrm{T}_{3}$ presentó promedios similares $(\mathrm{a}, \mathrm{b}, \mathrm{c}) \mathrm{a} \mathrm{T}_{1}$ y $\mathrm{T}_{2^{\prime}}$ principalmente, a los tres y seis meses de muestreo (Figura 2b).

A pesar de los resultados de los análisis estadísticos (Figura 2a), hubo cambios en la cobertura, donde a mayor proporción de compost, se presentaron las coberturas superiores, siendo $\mathrm{T}_{3}$ el que alcanzó los valores más altos durante los nueve meses de seguimiento, seguido de $T_{2}, T_{1}$ y control, en el cual se registraron muy bajas coberturas, menores al uno por ciento, a los nueve meses.

El seguimiento de la sucesión vegetal mostró que el incremento en altura de las especies fue proporcional a la cantidad de compost aplicado,
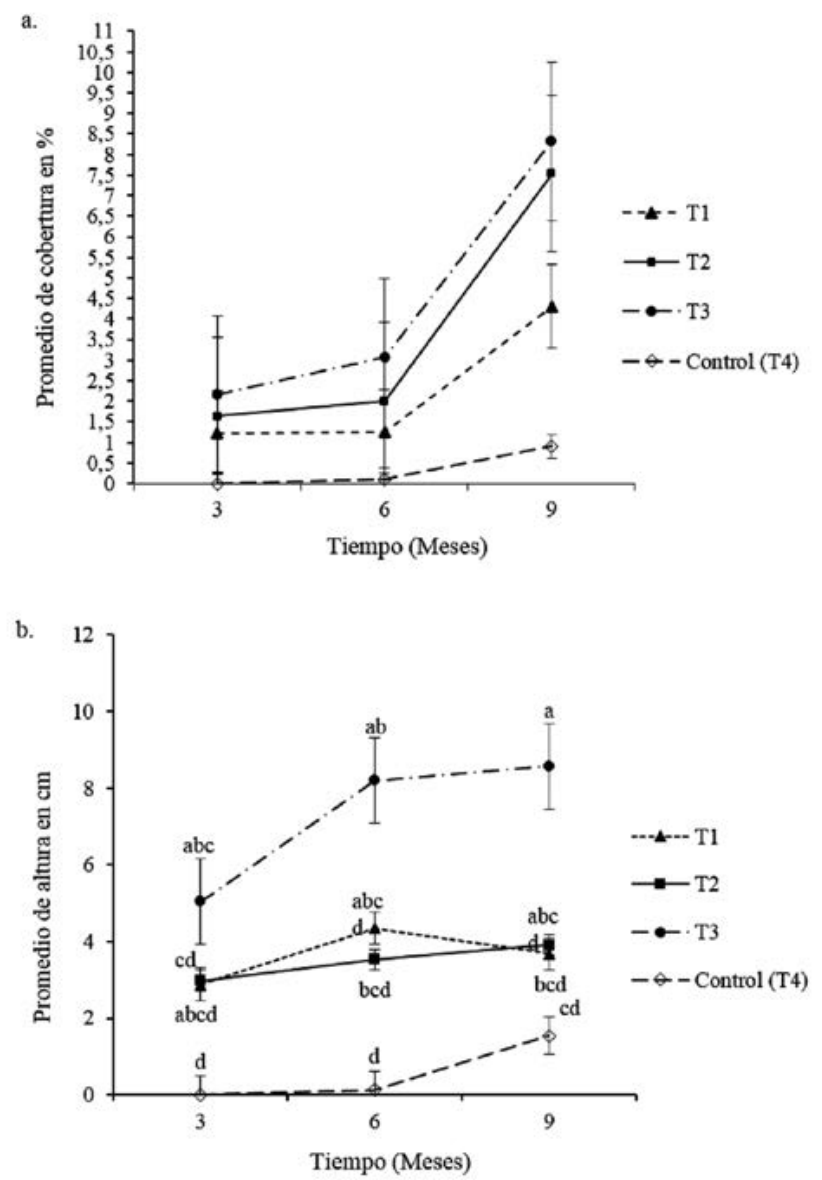

Figura 2. a) Promedio de cobertura en porcentaje (\%) de las especies en los tratamientos y el control, durante los nueve meses de seguimiento a la sucesión; b) Promedio de la altura en centímetros $(\mathrm{cm})$ de las especies en los tratamientos y el control. Las letras (a, b, c y d) indican diferencias entre los promedios de los tratamientos durante los nueve meses de seguimiento a la sucesión. 
de manera que $\mathrm{T}_{3}$ presentó los mayores valores, seguido de $T_{2}$ y $T_{1}$ a los tres (M1), seis (M2) y nueve meses (M3), y $\mathrm{T}_{4}$ presentó las plantas de menor tamaño (Tabla 3).

\section{Caracterización físico-química del sustrato al inicio y final del seguimiento}

La comparación de los parámetros físico-químicos del inicio y el final del seguimiento mostró que en $\mathrm{T}_{1}$ aumentó el valor de ocho características, entre las que sobresalen: $\mathrm{Ca}+\mathrm{Mg} / \mathrm{K}$, que varió de 9.7 a $10.1 \mathrm{meq} / 100 \mathrm{~g}$, el Mg/K de 1.96 a $2.25 \mathrm{meq} / 100 \mathrm{~g}$ y la materia orgánica $1.97 \%$ a $2.05 \%$; disminuyó el valor de 10 características, entre estas el pH (6.7 a 6.3) y el Ca, que varió de 13.7 a 12.1 meq.100g de sustrato; mientras la arcilla (33\%), el aluminio $(0 \%)$ y la textura (franco arcilloso) se mantuvieron iguales. En el $\mathrm{T}_{2}$ aumentó el valor de cuatro características, entre estas $\mathrm{Ca}+\mathrm{Mg} / \mathrm{K}$ (4.44 a 7.61 meq/100g) y el $\mathrm{pH}$ (7.0 a 7.3); se redujo el valor de 12 características, como Ca (15.6 a 10.3 meq.100g de sustrato), el fósforo disponible o Bray II (132 a $83.2 \mathrm{ppm}$ ) y el porcentaje de materia orgánica (3.78 a 1.91); y se mantuvo igual el valor de cinco características, entre estas: arcilla (29\%), arena (47\%) y limo (24\%). En el $\mathrm{T}_{3}$ aumentaron cinco características, entre las que se destacan $\mathrm{Mg} / \mathrm{K}$, que cambió de 0.68 a $1.81 \mathrm{meq} / 100 \mathrm{~g}$, el pH (7.4 a 8.0) y el limo (20 a $24 \%$ ); disminuyó el valor de 13 parámetros, como Ca (18.1 a 12.7 meq/100g de sustrato) y el K (5.11 a 2.08 meq/100g de suelo); en este tratamiento, la textura (franco arcilloso arenoso) y el aluminio (0\%) se mantuvieron iguales. En el control $\left(\mathrm{T}_{4}\right)$ aumentó el valor de 12 características, de las cuales se destacan el cambio de $\mathrm{Ca}+\mathrm{Mg} / \mathrm{K}$ de 11.7 a $19.7 \mathrm{meq} / 100 \mathrm{~g}$, el porcentaje de materia orgánica (0.22 a 0.62) y el limo (20 a 26\%); descendió el valor de siete parámetros, como el aluminio $(1.0$ a 0 meq $/ 100 \mathrm{~g}$ de sustrato), la arena (39 a 35\%) y Ca/Mg (1.55 a 1.22 me$\mathrm{q} / 100 \mathrm{~g}$; mientras se conservaron la textura (franco arcilloso) y la arena (39\%) del inicio y final del seguimiento.

El análisis de componentes principales (ACP) explicó un $76.87 \%$ de variabilidad (Figura 3), donde el componente 1 explicó la mayor parte de la varianza $(65.9 \%)$, dada principalmente por las variables CICE, fósforo disponible (Bray II), $\mathrm{pH}$ y la $\mathrm{MO}$, presentes principalmente en los tratamientos

Tabla 3. Altura $(\mathrm{cm})$ de las especies \pm el error estándar, en cada tratamiento y el control durante los nueve meses de seguimiento. T: Tratamiento, M: Muestreo.

\begin{tabular}{|c|c|c|c|c|c|c|c|c|c|c|c|c|}
\hline \multirow[t]{2}{*}{ Especie } & \multicolumn{3}{|c|}{$T_{1}$} & \multicolumn{3}{|c|}{$\mathbf{T}_{2}$} & \multicolumn{3}{|c|}{$\mathbf{T}_{3}$} & \multicolumn{3}{|c|}{$\mathbf{T}_{4}$} \\
\hline & M1 & M2 & M3 & M1 & M2 & M3 & M1 & M2 & M3 & M1 & M2 & M3 \\
\hline A. decurrens & $2.0 \pm 0.22$ & - & - & $2.6 \pm 0.04$ & 3 & - & 3 & $4.8 \pm 0.52$ & $8.7 \pm 0.15$ & - & - & - \\
\hline B. triplinervia & $1.3 \pm 0.15$ & $1.4 \pm 0.26$ & - & 2 & - & - & - & - & - & - & - & - \\
\hline Bidens sp. & - & 3 & $2.6 \pm 0.45$ & - & - & 3 & - & - & $5.3 \pm 0.15$ & - & 0.5 & $2.7 \pm 0.15$ \\
\hline C. petiolare & $7.7 \pm 0.78$ & $14.7 \pm 1.05$ & $20.5 \pm 0.13$ & $10.4 \pm 0.87$ & $16.8 \pm 1.19$ & $22.5 \pm 0.12$ & $9.6 \pm 0.68$ & $16.4 \pm 1.59$ & $23 \pm 0.2$ & - & - & - \\
\hline Chenopodium sp. & $16.8 \pm 1.42$ & $26.8 \pm 0.09$ & - & $11.8 \pm 2.19$ & $9.5 \pm 1.79$ & - & $22.7 \pm 2.19$ & $35.3 \pm 0.44$ & $3.3 \pm 0.15$ & - & - & - \\
\hline H. radicata & - & - & - & 1 & $6.3 \pm 0.44$ & $6 \pm 0.53$ & - & - & - & - & 1 & $1.3 \pm 0.12$ \\
\hline M. parviflora & - & - & - & - & - & - & $8 \pm 0.46$ & $24.7 \pm 3.62$ & $38.7 \pm 0.31$ & - & - & - \\
\hline Medicago sp. & $1.6 \pm 0.09$ & $3.2 \pm 2.88$ & 4 & 2 & - & - & - & - & - & - & - & - \\
\hline P. clandestinum & $3.4 \pm 0.10$ & $3.1 \pm 0.18$ & 2 & $4.9 \pm 0.18$ & $10.2 \pm 1.04$ & $7.7 \pm 1.46$ & 6 & $16.3 \pm 2.59$ & $15.4 \pm 1.05$ & - & - & $1.4 \pm 0.09$ \\
\hline P. annua & $5.1 \pm 0.42$ & $9.1 \pm 1.06$ & $16.6 \pm 0.15$ & $4.6 \pm 0.04$ & $10 \pm 1.86$ & $18.7 \pm 0.55$ & $6 \pm 0.53$ & - & - & $\therefore$ & - & $11.3 \pm 2.26$ \\
\hline P. aviculare & - & - & - & - & - & - & $14.3 \pm 0.67$ & $16.3 \pm 0.15$ & $17 \pm 0.15$ & - & - & - \\
\hline P. linearis & - & - & - & - & - & - & - & - & - & - & 0.5 & $3.9 \pm 0.27$ \\
\hline S. oleraceus & $1.1 \pm 0.06$ & 4 & $8 \pm 2.54$ & $1.4 \pm 0.09$ & 1.6 & 0.8 & $4.8 \pm 1.01$ & $8.3 \pm 0.39$ & $18 \pm 0.70$ & - & - & - \\
\hline
\end{tabular}




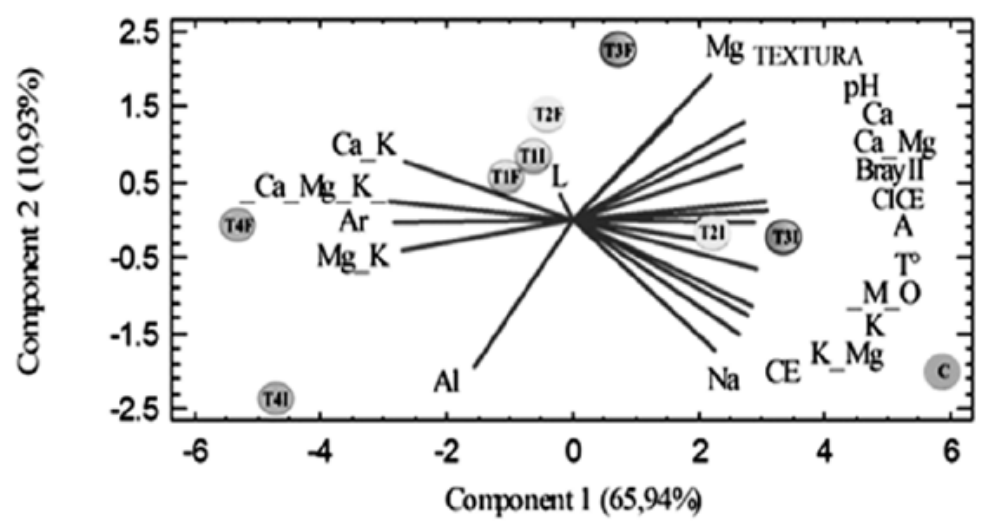

Figura 3. Análisis de componentes principales (ACP) de las características físico-químicas del sustrato al inicio y final del experimento. T1l y T1F (tratamiento 1 inicial y final); T2I y T2F (tratamiento 2 inicial y final); T3I y T3F (tratamiento 3 inicial y final), T4I y T4F (control inicial y final) y C (compost).

con mayor proporción de compost $(\mathrm{C})$, mientras el control $\mathrm{T}_{4}$ está influenciado por las variables $\mathrm{Al}$, $\mathrm{Mg} / \mathrm{K}$ y $\mathrm{Ca} / \mathrm{K}$, principalmente. La ubicación de $\mathrm{T}_{2}$ y $\mathrm{T}_{3}$ al final estaría relacionada con disminución de algunos componentes del compost.

El análisis mostró cambios de los parámetros físico-químicos evaluados en los tratamientos y el control a través del tiempo que permiten valorar las características del compost con respecto a los tratamientos y el control, al observar que el uso de esta enmienda orgánica redujo principalmente los niveles de $\mathrm{Al}, \mathrm{Ca} / \mathrm{K}$ y $\mathrm{Ca}+\mathrm{Mg} / \mathrm{K}$ en el estéril y el aumento de 14 parámetros de las mezclas estéril compost que probablemente favorecieron la llegada de especies vegetales a los tres meses de su aplicación.

\section{DISCUSIÓN}

\section{Cambios en la composición florística de los tratamientos y el control}

Las especies que crecieron en las parcelas fueron del estrato rasante y herbáceo, según los rangos descritos por Rangel \& Lozano (1986), y que concuerda con lo reportado por Ochoa \& Barrera
(2007), quienes registraron la colonización inicial de especies herbáceas, generalmente ruderales y competitivas que explotan mejor los hábitats de fertilidad moderada a alta (Chapin, 1980; Fuentes et al., 2006), lo que supone que la aplicación de compost mejora la fertilidad y fomenta el desarroIlo de la vegetación herbácea en áreas disturbadas por actividades mineras (Rondón \& Vidal, 2005; Kowaljow \& Mazzarino, 2007; Farrell et al., 2011).

Las familias Asteraceae y Poaceae fueron las más abundantes en las parcelas experimentales, esto coincide con los estudios realizados por Rozo (2007), Ochoa \& Barrera (2007), quienes utilizaron biosólidos como enmienda orgánica, lo cual muestra que las especies de estas familias Ilegan primero gracias a la dispersión anemócora de sus semillas y que son muy abundantes gracias a su ciclo de vida corto, características que las hacen especies pioneras (Lozano et al., 2008); además Mendoza \& Ramírez (2000) encontraron que la mayoría de especies de la familia Asteraceae y Poaceae pueden Ilegar fácilmente a zonas abiertas de ambientes secos y vegetación de baja altura, lejos de las plantas parentales.

Las condiciones climáticas afectaron los cambios sucesionales de la vegetación en las parcelas experimentales a través del tiempo. En los 
primeros meses (diciembre, enero, febrero) hubo altas temperaturas, menor humedad y precipitación, factores que afectaron la germinación de algunas especies; en los meses siguientes (abril, mayo, junio) disminuyó la temperatura, aumentaron las precipitaciones y la presencia de avifauna, que favorecieron la llegada de especies y el aumento de cobertura vegetal, mientras en el último periodo de muestreo (julio, agosto y septiembre) fluctuaron las condiciones climáticas, la temperatura y las precipitaciones se redujeron, y ocasionaron la pérdida de especies vegetales en las parcelas experimentales (Figura 4); los aspectos climáticos definieron la colonización y la tasa de supervivencia de las plántulas, los cuales han sido destacados en los procesos sucesionales por Connell \& Slatyer (1977), Peet \& Christensen (1980) y Huston \& Smith (1987).
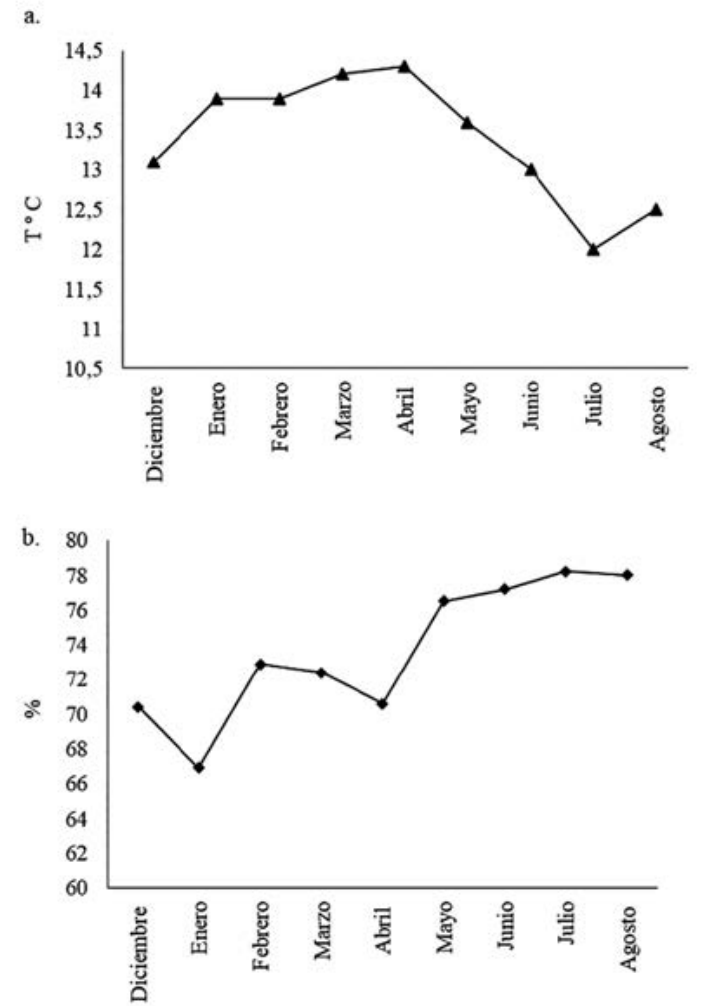

\section{Diversidad en los tratamientos y el control}

Es importante medir la diversidad en áreas afectadas por minería a cielo abierto, ya que permite cuantificar el avance de los procesos sucesionales y la recuperación de la estructura y composición de las áreas afectadas por este disturbio (Barrera \& Valdés, 2007; Barrera et al., 2007). En este estudio, los aumentos de diversidad en los tratamientos fueron significativos con respectos al control T4, ya que los resultados de riqueza específica, índice de Shannon e índice de Simpson mostraron la efectividad de la aplicación de enmiendas orgánicas, en este caso compost, sobre el aumento de la diversidad y la composición vegetal de áreas altamente disturbadas, como lo indica Ochoa \& Barrera (2007), Burgos et al. (2007) y Miranda (2009)

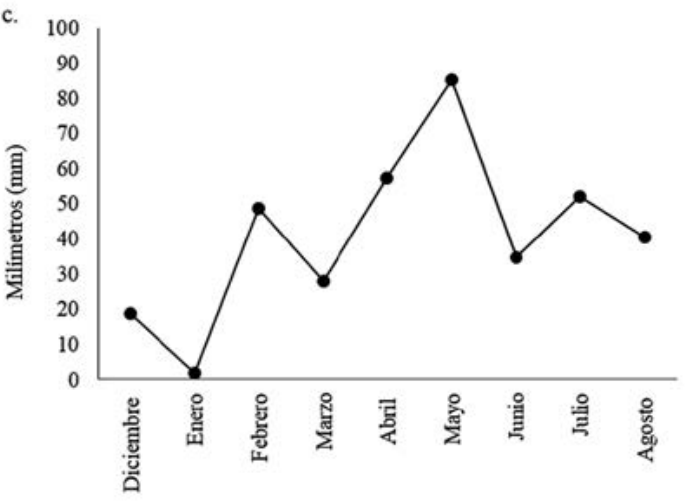

Figura 4. Variaciones climáticas durante los meses de muestreo, a) Temperatura ambiente $\left({ }^{\circ} \mathrm{C}\right)$, b) Humedad relativa (\%), c) Precipitación (mm). 
El tratamiento 3 estimuló más el aumento de la diversidad, mientras $T_{1}, T_{2}$ y el control presentaron una menor diversidad al final del seguimiento, quizás porque en $\mathrm{T}_{3}$ la materia orgánica fue mayor y facilitó la infiltración y regulación hídrica que permitieron un mejor desarrollo y sobrevivencia de las especies vegetales. En contraste, Rozo (2007), Ochoa \& Barrera (2007) registraron una mayor diversidad en el tratamiento 1 , con menor proporción de enmienda. La riqueza específica de $\mathrm{T}_{3}$ aumentó progresivamente, y llegó a ser mayor que la reportada por Rozo (2007), Ochoa \& Barrera (2007), donde $T_{3}$ presentó un menor número de especies, mientras en T1 encontraron el mayor número de especies. En el último muestreo, $\mathrm{T}_{3}$ mostró mayor número de especies que el control $\left(\mathrm{T}_{4}\right)$, lo que indica que la aplicación de enmiendas orgánicas en áreas afectadas por actividades extractivas mejora la disponibilidad de nutrientes (Tabla 1) y el crecimiento de las plántulas (Zmora-Nahum et al., 2005).

Los tratamientos 2 y 3 tienen igual número de especies, pero $\mathrm{T}_{3}$ tiene mayor diversidad, lo que muestra que la alta disponibilidad de nutrientes probablemente mejora la composición de la vegetación, en lo que coincide Terradas (2001); al contrario, Grime (1973), Martínez et al. (2003) y Rowe et al. (2006) registraron que los suelos fertilizados tienen una disminución de la diversidad de especies.

\section{Análisis de varianza (ANOVA) para la cobertura y altura}

El aumento de la cobertura durante el tiempo de seguimiento fue directamente proporcional a la cantidad de compost aplicado, siendo $\mathrm{T}_{3}$ el tratamiento que presentó los mejores resultados, lo que contrasta con la experiencia de Rozo (2007), Ochoa \& Barrera (2007), quienes encontraron que la mayor proporción de biosólido no influyó sobre el porcentaje de cobertura de las especies.

El análisis de varianza no mostró diferencias significativas de la cobertura entre tratamientos durante el tiempo evaluado, sin embargo, son importantes los aumentos de porcentajes de coberturas (Figura 2a), donde se observó que, a mayor cantidad de compost aplicado, existió un mayor porcentaje de cobertura vegetal, tal como lo registraron Kowaljow \& Mazzarino (2007) en un estudio realizado en un área semiárida de la Patagonia donde utilizaron compost de residuos municipales. Se puede decir que el compost sirve para recuperar coberturas vegetales y que probablemente estas ayuden a disminuir la erosión, mejorar la infiltración y retener agua en áreas afectadas por explotaciones mineras a cielo abierto, como lo ha sugerido Miranda (2009).

Respecto a la altura y cobertura de las especies, se estableció que el género Chenopodium se destacó por presentar el mayor porcentaje de cobertura y altura en los tratamientos con compost durante los meses evaluados, esto quizás se presentó porque la mayoría de especies de este género son heliófilas de rápido crecimiento y poseedoras de altas tasas fotosintéticas (Terradas, 2001), además son capaces de establecerse en áreas afectadas por actividades extractivas. Pennisetum clandestinum presentó altos porcentajes de cobertura y altura especialmente en los tratamientos con compost durante los meses de muestreo, debido a que es la especie dominante en el pastizal de los alrededores al montaje experimental y también por ser una especie perenne que crece a bajas temperaturas y tener la capacidad para invadir cualquier zona donde las condiciones de luz, humedad y materia orgánica sean favorables para la germinación de sus semillas (Labrada \& Parker, 1996; Ochoa \& Barrera, 2007).

Los análisis de varianza registraron diferencias significativas de la altura entre tratamientos, donde $T_{3}$ tuvo las mayores alturas, en contraste con el control, que registró alturas muy bajas de las especies durante todo el periodo de seguimiento. Esto muestra que la aplicación de compost favorece el crecimiento de las especies, como lo mencionaron García et al. (2002), Zmora-Nahum et al. (2005), Farrell et al. (2011) y Sizmur et al. (2011). 
Entonces, el restablecimiento de coberturas vegetales con ayuda de enmiendas orgánicas (compost) en áreas disturbadas por minería a cielo abierto es un paso inicial y fundamental para devolver parte de la estructura, funciones y servicios de los ecosistemas (regulación del microclima y el agua, el mejoramiento del hábitat y las propiedades del suelo), que promueve la llegada de otras especies vegetales y activa los procesos sucesionales hacia estados más avanzados, tal como lo indicaron Bradshaw (1997), Lubke \& Avis (1998), Ochoa \& Barrera (2007), Domene et al. (2011) y Farrell et al. (2011).

\section{Caracterización físico-química de los sustratos y el control al inicio y final del seguimiento}

Los cambios en las características físico-químicas del sustrato y la disponibilidad de los recursos edáficos permitieron cambios en la composición de especies vegetales a través del tiempo, como lo indicaron Peet \& Christensen (1980) y Huston \& Smith (1987). Tal es el caso de Chenopodium petiolare, predominante en los tratamientos con compost, la cual es una especie con requerimientos elevados de nutrientes y luz, con una eficiente absorción de nutrientes por la raíz cuando hay alta disponibilidad de estos en el sustrato, características que favorecen su rápido crecimiento (Terradas, 2001); en contraste con el control $\left(T_{4}\right)$, que solo presentó algunas especies durante el tiempo de seguimiento. Dentro de las especies que aparecieron en el control estuvo Hypochaeris radicata, que es una especie caracterizada por presentar raíces gruesas y fuertes, capaces de crecer en suelos ácidos y poco fértiles (Dietl et al., 2009).

Los análisis físico-químicos del sustrato mostraron que la adición de compost proporcionó mayor cantidad de materia orgánica, disminuyó la acidez e incrementó la temperatura, los nutrientes y minerales esenciales para la germinación, crecimiento y supervivencia de las plantas, al regular la absorción y almacenamiento de agua, como lo indicaron Farrell et al. (2011) y Domene et al. (2011), factores que favorecieron la llegada de especies vegetales a las parcelas con tratamiento y su ausencia en las parcelas control; además, los análisis físico-químicos determinaron que las características en el $\mathrm{T}_{1}$ final presentaron un sustrato de $\mathrm{pH}$ neutro por efecto del compost, al reducirse los ácidos orgánicos y aumentar la producción de amonio (Beck-Friis, et al., 2003); también disminuyó la cantidad de materia orgánica, el contenido de nutrientes, además la baja infiltración, la impermeabilidad y la escorrentía del agua en este sustrato probablemente condicionaron el establecimiento de las especies vegetales, (García et al., 2009). El T al final registró un sustrato ligeramente ácido con bajo contenido de materia orgánica, sin embargo la salinidad fue relativamente alta, por el aumento de $\mathrm{Ca}$, Mg y $\mathrm{K}$ aportados por el compost y también por los cambios en el balance hídrico (Jobbásy et al., 2008), especialmente la disminución de los niveles de precipitación, factores que posiblemente determinaron el establecimiento de las plantas en el sustrato, ya que algunas especies toleran más sales que otras (López \& López, 1978) y la acidez limita el crecimiento y el desarrollo de las raíces (Pritchett, 1986).

El $\mathrm{T}_{3}$ presentó los contenidos más altos de $\mathrm{P}$, porque presentó la mayor proporción de compost que es rico en este nutriente (Tabla 1). Según Terradas (2001), la presencia de P al inicio de la sucesión es importante para liberar los procesos de captación de $\mathrm{N}$, requerido por todas las especies para su crecimiento, el que efectivamente fue mayor en $\mathrm{T}_{3}$, como se mencionó, frente al control y los demás tratamientos. En la sucesión primaria, el $\mathrm{P}$ se libera como fosfatos por la acción del agua y los ácidos, (Terradas, 2001), pero en el control $\left(\mathrm{T}_{4}\right)$ su cantidad es reducida (Tabla 1 ), y por esto el crecimiento de las especies es bajo, como se mostró en la Tabla 3. Además, en el control al inicio y final se observaron valores bajos de materia orgánica y $\mathrm{pH}$ moderadamente ácido por el alto contenido de aluminio y los bajos niveles de nutrientes que dificultaron el establecimiento de las plantas, 
como lo indicaron Pritchett (1986), Gensemer \& Prayle (1999), Pichtel \& Bradway (2008), y por esto se retardó la recuperación de la cobertura vegetal en las parcelas sin compost, como ocurre en áreas disturbadas por actividades extractivas a cielo abierto, lo cual fue descrito por Lubke \& Avis (1998) y Andrés (2009).

\section{CONCLUSIONES}

La aplicación de la mezcla estéril/compost en proporción 2:1 $\left(\mathrm{T}_{3}\right)$ fue la más eficaz para el restablecimiento de coberturas vegetales, aumento de la altura y la diversidad de especies; del mismo modo, esta mezcla tiene las características físico-químicas que facilitaron el mejor establecimiento y desarrollo de las especies en las parcelas con tratamiento.

El compost demostró ser una enmienda orgánica efectiva en la inducción de la sucesión vegetal y la recuperación de la vegetación en las áreas disturbadas por actividades extractivas a cielo abierto, ya que en menor tiempo los tratamientos con compost presentaron mejores resultados frente al control.

\section{AGRADECIMIENTOS}

Este estudio fue realizado gracias al apoyo financiero de la Dirección de Investigaciones (DIN) de la Universidad Pedagógica y Tecnológica de Colombia y al ingeniero Jaime Moreno, gerente de la Ladrillera Fénix, Tunja S.A.S. Agradecemos a Daniel Galindo por la asesoría en los análisis estadísticos, a Beatriz Miranda Mojica por las observaciones al trabajo de investigación, a Manuel Galvis por la colaboración en la determinación de las especies vegetales y a los integrantes del grupo de investigación Ecología de Bosques Andinos Colombianos (EBAC) por sus aportes durante la elaboración del artículo.

\section{REFERENCIAS BIBLIOGRÁFICAS}

Aguilar, M. (2009). El papel de las caracterizaciones diagnósticas en la restauración ecológica de áreas degradadas por minería a cielo abierto. En: J.I. Barrera, S. Contreras, A.C. Ochoa, S. Perilla, N. Garzón \& D. Rondón, (eds.). Restauración ecológica de áreas degradadas por minería a cielo abierto (pp. 104-116). Bogotá D.C.: Editorial Pontificia Universidad Javeriana.

Andrés, P. (2009). La restauración ecológica: objetivos y aspectos generales. En: J.I. Barrera, S. Contreras, A.C. Ochoa, S. Perilla, N. Garzón \& D. Rondón (eds.). Restauración ecológica de áreas degradadas por minería a cielo abierto (pp. 21-33). Bogotá D.C.: Editorial Pontificia Universidad Javeriana.

Barrera, J.I. (2009). Barreras al restablecimiento natural y a la restauración ecológica de áreas afectadas por minería a cielo abierto (pp. 35-44). En: J.I. Barrera, S. Contreras, A.C. Ochoa, S. Perilla, N. Garzón \& D. Rondón (eds.). Restauración ecológica de áreas degradadas por minería a cielo abierto. Bogotá D.C.: Pontificia Universidad Javeriana.

Barrera, J.I., \& Valdés, C. (2007). Herramientas para abordar la restauración ecológica de áreas disturbadas en Colombia. Universitas Scientiarum, 12 (2), 11-24.

Barrera, J.I., Campos, C., \& Montoya, S. (2007). Experiencia piloto de restauración ecológica en la cantera Soratama, mediante el uso de biosólidos como enmienda orgánica. Universitas Scientiarum, 12, 5-11.

Barrera, J.I., Contreras, S.M., Garzón, N.B., \& Moreno, A.C. (2010). (eds.). Manual para la restauración ecológica de los ecosistemas disturbados del Distrito Capital. Bogotá D.C.: Pontificia Universidad Javeriana. $401 \mathrm{p}$.

Beck-Friis, B., Smårs, S., Jönsson, H., Eklind, Y. \& Kirchmann, H. (2003). Composting of source-separated household organics at different oxygen levels: Gaining an understanding of the emission dynamics. Compost Science \& Utilization 11, 41-50.

Braun-Blanquet, J.(1964). Pflanzensoziologie: Grundzüge der Vegetationskunde. New York: Editorial Springer. 865 p. 
Bradshaw, A. (1997). Restoration of mined lands-using natural processes. Ecological Engineering., 8, 255-269.

Burgos, P., Pérez De Mora, A., Madejon, E., \& Cabrera, F. (2007). Aplicación de enmiendas para la recuperación de la vegetación espontánea de un suelo contaminado con elementos traza. Tendencias Actuales de la Ciencia del Suelo, 228-235.

Chapin, F.S. (1980). The mineral nutrition of wild plants. Annual Review of Ecology and Systematics, 11, 233-260.

Connell, J.H., \& Slatyer, R.O. (1977). Mechanisms of succession in natural communities and their role in community stability and organization. The American Naturalist, 111, 1119-1144.

Compañía de Servicios Públicos de Sogamoso-COSERVICIOS S.A. ESP. (2007). Guía práctica para el manejo de residuos sólidos. Sogamoso: Coservicios, Alcaldía Municipal de Sogamoso. 28 p.

Dietl, W., Fernández, F., \& Venegas C. (2009). Manejo sostenible de praderas, su flora y vegetación. Chile: Ministerio de Agricultura, ODEPA. 188 p.

Domene, X., Solá, L., Ramírez, W., Alcañiz, J., \& Andrés, P. (2011). Soil bioassays as tools for sludge compost quality assessment. Waste Management, 31, 512-522.

Etter, A., McAlpine, C., Wilson, K., Phinn, S., \& Possingham, H. (2006). Regional patterns of agricultural land use and deforestation in Colombia. Agriculture, Ecosystems and Environment, 114, 369-386.

Farrell, M., Perkins, W.T., Hobbs, P.J., Griffith, G.W., \& Jones, D.L. (2011). Migration of heavy metals in soil as influenced by compost amendments. Environmental Pollution, 158, 55-64.

Fuentes, C., Fúquene, A., Perdomo, E, \& Pinto, S. (2006). (eds.). Plántulas de especies arvenses frecuentes en la zona centro de Colombia. Bogotá D.C.: Editorial Universidad Nacional de Colombia. 248 p.

García, A., Mejuto, Del Riego, L., Cardona, A., Díaz, F., \& Rodríguez, V. (2009). Activación de los procesos de edafización en la rehabilitación de zonas degradadas por actividades mineras. Un caso de estudio. Water Soil Vegetation, 539-542.
García, A., Bernal, M.P., \& Roig, A. (2002). Growth of ornamental plants in two compost prepared from agroindustrial wastes. Bioresource Technology, 83(2), 81-87.

Gensemer, R. W. \& Playle, R.C. (1999). The bioavailability and toxicity of Aluminium in aquatic environments. Critical Reviews in Environmental Science and Technology, 29 (4), 315-450.

Grime, J.P. (1973). Control of species density in herbaceous vegetation. Environmental Management, 1, 151-167.

Gutiérrez, H., \& De La Vara, R. (2012). Análisis y diseño de experimentos. México: Editorial McGraw-HiII. 489 p.

Huston, M., Smith, T. (1987). Plant succession: life history and competition. American Naturalist, 130 (2), 169-199.

Jobbágy, E. , Nosetto, M., Santoni, C. Baldi, G. (2008). El desafío ecohidrológico de las transiciones entre sistemas leñosos y herbáceos en la Ilanura Chaco-Pampeana. Ecología Austral, 18, 305-322.

Kowaljow, A. E., \& Mazzarino, M. (2007). Soil restoration in semiarid Patagonia: Chemical and biological response to different compost quality. Soil Biology \& Biochemistry, 39, 1580-1588.

Labrada, R., Parker, C. (1996). Manejo de malezas para países en desarrollo (Estudio FAO producción y protección vegetal). Roma: Editorial Organización de las Naciones Unidas para la Agricultura y la Alimentación. 403 p.

López, R.J., \& López, M .J. (1978). El diagnóstico de suelos y plantas, métodos de campo y laboratorio. Madrid: Editorial Mundiprensa. 333 p.

Lozano, P., Bussman, R., Kupers, M., \& Lozano, D. (2008). Deslizamientos naturales y comunidades pioneras de ecosistemas montanos al occidente del Parque Nacional Podocarpus (Ecuador). Caldasia, 30 (1), 1-19.

Lubke, R.A., \& Avis, A.M. (1998). A Review of the concepts and application of rehabilitation following heavy mineral dune mining. Marine Pollution BuIletin, 37 (12), 546-557.

Martínez, F., Cuevas, G., Calvo, R., \& Walter, I. (2003). Biowaste effects and native plants in a semiarid ecosystem. Journal of Environmental Quality, 32, 472-479. 
Mendoza, H. C. \& Ramírez, B. (2000). Plantas con flores de la Planada. Guía ilustrada de familias y géneros. Bogotá D.C: Editorial Instituto de Recursos Biológicos Alexander von Humboldt. 244 p.

Miranda, B. T. (2009). Estrategias para la restauración del componente suelo en áreas afectadas por minería (pp. 161-173). En: J.I. Barrera, S. Contreras, A.C. Ochoa, S. Perilla, N. Garzón N, \& D. Rondón (eds.). Restauración ecológica de áreas degradadas por minería a cielo abierto. Bogotá D.C.: Editorial Pontificia Universidad Javeriana.

Ministerio de Minas y Energía. (2003). Glosario Técnico Minero. República de Colombia. Bogotá D.C. Ministerio de Minas y Energía. 168 p.

Müeller, D, \& Ellenberg, H, (1974). Aims and methods of vegetation ecology. Estados Unidos: Editorial Library of Congress Cataloging. 547 p.

Negrete, R.E. (2013). Derechos, minería y conflictos. Aspectos normativos. En: L.J .Garay (ed.) Minería en Colombia: fundamentos para superar el modelo extractivista (pp. 23-54). Bogotá D.C.: Editorial Contraloría General de la República de Colombia.

Neil Polhemus(1980). STATGRAPHICS 5.1 PLUS. Warrengton: Statpoint Technologies Inc. Recuperado de: http://www.statgraphics.com/

Ochoa, A., \& Barrera, J.I. (2007). Efecto de la aplicación de biosólidos, sobre el desarrollo de la vegetación en las primeras etapas sucesionales en la cantera Soratama, localidad de Usaquén, Bogotá. Universitas Scientiarum, 12 (2), 57-72.

Peet, R. K., \& Christensen, N.L. (1980). Succession: a population process. Vegetation, 43, 131-140.

Plan Nacional de Restauración (PNR). (2012). Restauración ecológica, rehabilitación y recuperación de áreas disturbadas. Ministerio de Ambiente y Desarrollo Sostenible. Bogotá D.C. 86 p.

Pritchett, W. (1986). Suelos forestales. Propiedades, conservación y mejoramiento. México: Editorial Limusa. 634 p.

Pichtel, J., \& Bradway, D.J. (2008). Conventional crops and organic amendments for $\mathrm{Pb}, \mathrm{Cd}$ and $\mathrm{Zn}$ treatment at a severely contaminated site. Bioresource Technology 99, 1242-1251.
Polhemus, N. (1980). STATGRAPHIC 5.1. Virginia. Statistics package: that performs and explains basic and advanced statistical functions. Descargado de: http:// statgraphics.softnic.com/.

Ramírez, A, (2006). Ecología, métodos de muestreo y análisis de poblaciones y comunidades. Bogotá D.C: Pontificia Universidad Javeriana. 271 p.

Rangel, J.O., \& Lozano, G. (1986;). Un perfil de vegetación entre La Plata (Huila) y el Volcán Puracé. Caldasia, 14(68-70), 53-547.

Rondón, J., \& Vidal, R. (2005). Establecimiento de la cubierta vegetal en áreas degradadas (Principios y Métodos). Forest Latin, 38, 63- 82.

Rowe, E.C., Healey, J.R., Edwards-Jones, G., Hills, J., Howells, M., \& Jones, D.L. (2006). Fertilizer application during primary succession changes the structure of plant and herbivore communities. Biological Conservation, 131, 510-522.

Rozo, N.A. (2007). Efecto de la aplicación de mezclas de biosólidos y estériles sobre las primeras etapas de la sucesión en la antigua arenera Juan Rey, Bogotá D.C. (Trabajo de pregrado, Ecología). Bogotá D.C.: Pontificia Universidad Javeriana. 92 p.

Society For Ecological Restoration Internacional (SER). (2004). Principios del SER internacional sobre la restauración ecológica. Grupo de trabajo sobre ciencia y políticas, Tucson, Arizona.

Sizmur, T., Wingate, J., Hutchings, T., Hodson, $M$. (2011). Lumbricus terrestris L. does not impact on the remediation efficiency of compost and biochar amendments. Pedobiología, 54 (5), 211-216.

Terradas, J. (2001). Ecología de la vegetación, de la ecofisiología de las plantas a la dinámica de comunidades y paisajes. Barcelona: Editorial Omega. $703 \mathrm{p}$.

Villas-Boas, R.C., \& Page, R. (2001) (eds). La minería en el contexto de la ordenación del territorio. Rio de Janeiro: Editorial Cooperación Iberoamericana. $418 \mathrm{p}$.

Zmora-Nahum, S., Markovitch, O., Tarchitzky, J., \& Chen, Y. (2005). Dissolved organic carbon (DOC) as a parameter of compost maturity. Soil Biology and Biochemistry, 37, 2109-2116. 